\title{
Neoadjuvant and adjuvant systemic treatment for hepatocellular carcinoma
}

\author{
Maria Cecilia Mathias-Machado, Leonardo G. da Fonseca \\ Clinical Oncology, Instituto do Cancer do Estado de Sao Paulo, University of Sao Paulo, São Paulo 01246-000, Brazil. \\ Correspondence to: Dr. Leonardo G. da Fonseca, São Paulo Clínicas Liver Cancer Group - Hospital das Clínicas Complex, \\ Instituto do Cancer do Estado de São Paulo, University of São Paulo School of Medicine. Av Dr. Arnaldo, 450, São Paulo 01246- \\ 000, Brazil. E-mail: I.fonseca@fm.usp.br
}

How to cite this article: Mathias-Machado MC, da Fonseca LG. Neoadjuvant and adjuvant systemic treatment for hepatocellular carcinoma. Hepatoma Res 2021;7:67. https://dx.doi.org/10.20517/2394-5079.2021.84

Received: 25 Jun 2021 First Decision: 4 Aug 2021 Revised: 9 Aug 2021 Accepted: 23 Aug 2021 Published: 15 Oct 2021

Academic Editors: James Fung, Allan Tsung Copy Editor: Yue-Yue Zhang Production Editor: Yue-Yue Zhang

\begin{abstract}
Hepatocellular carcinoma (HCC) is a highly lethal malignancy, and few patients are candidates for curativeintended therapies. The mainstay of curative treatment in HCC is surgical resection, ablation, and transplantation. However, rates of recurrence are high, and there is no established approach to reduce the risk of recurrence and mortality. We discuss the available data and current landscape of (neo)adjuvant therapies aimed at decreasing recurrence risk and improving overall survival, including liver-directed therapies, tyrosine kinase inhibitors, and immunotherapy. Neoadjuvant strategies aimed at downstaging advanced HCC to enable local treatment and minimize the risk of recurrence using novel agents are also a topic of interest in current research. The improvements achieved in the advanced stages with immune-checkpoint inhibitors are priming ongoing trials that address potential future directions for both adjuvant and neoadjuvant strategies that may change the treatment paradigm of $\mathrm{HCC}$ in the near future.
\end{abstract}

Keywords: Liver neoplasm, hepatocellular carcinoma, neoadjuvant treatment, adjuvant treatment, immunotherapy

\section{INTRODUCTION}

Hepatocellular carcinoma (HCC) is a highly fatal disease, although several strategies for early diagnosis and management have emerged in recent years. Clinical treatment approaches are challenging due to the concomitance of HCC with cirrhosis complications and the fact that many patients are diagnosed at an 
advanced stage. In these cases, patients are not candidates for curative intent local treatments such as resection, percutaneous ablation, or liver transplantation ${ }^{[1]}$.

In patients diagnosed with early-stage disease, especially cases detected during screening, curative treatments are chosen in order to achieve a complete and durable remission. However, recurrence rates are high, reaching up to $70 \%$ in five years ${ }^{[2]}$. This scenario highlights the importance of strategies and treatments able to reduce the risk of recurrence and, consequently, impact positively on mortality.

Currently, there are no established treatments in the adjuvant setting post resection, ablation, or transplantation. The main treatment guidelines indicate that patients should remain under follow-up and additional treatment is not recommended. Accordingly, no neoadjuvant therapy has proven to reduce the risk of recurrence and is not part of treatment algorithms $\mathrm{s}^{[1,3]}$. The use of downstaging strategies for patients beyond criteria for liver transplantation can be considered one of the only exceptions.

Some data suggest benefit with the use of interferon-alpha (IFN $\alpha$ ), cytokine-induced killer cells, arterial chemoembolization, and antiviral therapies. However, robust evidence of the efficacy of such therapies is scarce.

Currently, new drugs have encouraging results in the treatment of advanced HCC, especially immune checkpoint inhibitors. As a result, several clinical trials are ongoing aiming at the incorporation of such agents to reduce risk of recurrence. The present review describes the rationale and available data on adjuvant treatment after local treatments in HCC.

\section{RATIONALE FOR (NEO)ADJUVANT TREATMENT}

Conceptually, HCC recurrence can occur in different settings: (1) intrahepatic recurrence; (2) de novo HCC; or (3) appearance of extrahepatic metastatic lesions with or without a liver tumor [Figure 1].

The first scenario occurs more frequently up to two years after local treatment, while the second scenario has a later onset (more than two years) and is the result of risk factors that predispose to cirrhosis-induced carcinogenesis. The third scenario, the appearance of extrahepatic metastatic lesions, is associated with poor prognosis ${ }^{[4]}$.

Some studies indicate that distinct genomic profiles between primary and recurrent HCC could explain poor prognosis of recurrence. Transcriptomic reprogramming of tumor cells with acquired mutations in specific genes, such as TP53 and ARID1A, are suggested to trigger clonal evolution and aggressive phenotypes with invasion, metastasis, and epithelial-to-mesenchymal transition ${ }^{[5,6]}$.

Recurrence prevention through adjuvant treatment is distinct in cases of de novo HCC. From this standpoint, the management of cirrhosis complications, as well as measures to avoid liver function deterioration through antiviral treatment, for example, are crucial. To reduce the risk of recurrence due to intrahepatic and extrahepatic metastases, the use of systemic therapies is promising.

The rational for neoadjuvant therapeutic approaches before local treatment is based on an early control of micrometastatic disease and the possibility of a tumor downsizing, which could improve the feasibility of resection or ablation. The use of immunotherapy is being increasingly investigated in this scenario. 


\section{Strategies}

\section{De novo hepatocelular carcinoma}

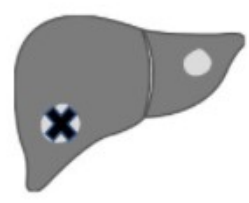

$>2$ years

Cirrhosis as risk factor

De novo carcinogenesis
Anti-viral treatment

Treatment for metabolic disorders

Alcohol cessation

\section{Hepatic metastasis}

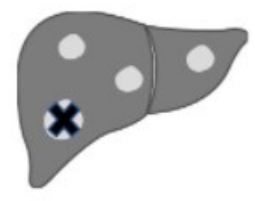
$><2$ years
$>$ Risk factors: size, number, grade, vascular invasion, margin

Clinical trials

Immunotherapy

\section{Extrahepatic metastasis}

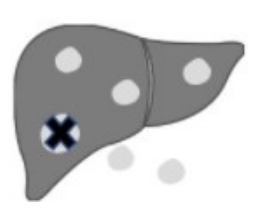

$<2$ years

Risk factors: size, number, grade, vascular invasion
Clinical trials

Immunotherapy

Figure 1. Patterns of recurrence after resection or ablation and potential strategies.

\section{SELECTION OF POTENTIAL CANDIDATES FOR ADJUVANT THERAPIES}

The risk of recurrence after resection, ablation, or transplantation can be determined by well-defined factors and other factors that are still unknown. Tumor-related characteristics such as size, number of nodules, vascular invasion (macroscopic or microscopic), or residual disease after incomplete resection are significantly related to higher recurrence rates. Genetic signatures, circulating microRNA, and circulating tumor cells have also been shown to predict the risk of recurrence and can become an important tool for patient selection ${ }^{[4,7]}$.

With the increasing awareness of the role of immunology in HCC, some immunological features are being considered as potential predictors of recurrence. For example, the concentration of intratumor $\mathrm{CD} 3+$ and $\mathrm{CD} 8+\mathrm{T}$ cells at tumor resection margins is associated with recurrence. Furthermore, the expression of programmed death ligand 1 (PD-L1) by immune and tumor cells is associated with tumor aggressiveness and risk of recurrence ${ }^{[8]}$.

Recognition of higher risk characteristics is crucial for clinical trial design and for the appropriate selection of target populations that may benefit from complementary treatment strategies.

\section{ADJUVANT TREATMENT MODALITIES}

IFNa

Some studies conducted in Asia with patients with chronic hepatitis B virus infection suggest that the use of IFN $\alpha$ may play a role in the adjuvant setting. In a randomized study involving 236 patients with hepatitis B 
virus infection who underwent surgical resection, the use of IFN $\alpha$ at five million units three times a week was associated with better overall survival (63.8 months vs. 38.8 months for the control group) and better recurrence-free survival (31.2 months $v s .17 .7$ months) ${ }^{[9]}$. In addition, a meta-analysis including 14 studies (9 randomized trials and 5 cohort studies) showed that IFN $\alpha$ is associated with a reduction in recurrence and mortality in patients infected with hepatitis $\mathrm{C}$ virus, while in patients with hepatitis $\mathrm{B}$ virus there was a reduction in mortality, but no impact on HCC recurrence was demonstrated ${ }^{[10]}$. Despite the mentioned data, the results with IFN $\alpha$ have not been globally reproduced, and this treatment is not considered a standard-ofcare according to recommendation guidelines ${ }^{[1,3]}$.

\section{Antiviral drugs}

The use of nucleotide/nucleoside analogs is recommended in patients with HCC and active hepatitis B virus infection. The effect of nucleotide/nucleoside analogs as an adjuvant therapy was evaluated in a longitudinal study with patients with chronic hepatitis B infection. This study showed lower rates of recurrence and deaths related to HCC, as well as higher probability of preserved liver function at six months after surgery ${ }^{[1]}$. The use of antiviral drugs is recommended for patients with chronic hepatitis B with detectable HBV-DNA and HCC.

\section{Intra-arterial treatment}

The use of transarterial chemoembolization (TACE) is the recommended treatment for patients with intermediate stage BCLC- $\mathrm{B}^{[1,3]}$. The rationale for using this modality as an adjuvant treatment stems from the potential benefit in detecting residual disease by angiography and early embolization of the residual tumor focus.

Two randomized studies with a limited number of patients suggest the role of TACE in the adjuvant setting. In the first study, patients with HCC associated with hepatitis B virus infection who underwent TACE after resection had favorable recurrence rates $(56 \% v$ s. $42.1 \%)$ and three-year survival rates $(85.2 \%$ vs. $77.4 \%)$ compared to the control group ${ }^{[12]}$. In another study, including patients with resected tumors larger than $5 \mathrm{~cm}$ or presenting microvascular invasion, the use of adjuvant TACE resulted in better overall survival (44.29 months vs. 22.37 months) when compared to the control $\operatorname{arm}^{[13]}$. However, randomized trials with a larger number of patients are warranted to refine the role of TACE after resection.

\section{Target therapies: tyrosine kinase inhibitors}

TKIs are essential tools for managing advanced HCC. Sorafenib, an inhibitor with activity against RAF and receptors for vascular growth factor-2 (VEGFR-2) and platelet-derived growth factor, was the first drug able to demonstrate a survival improvement with advanced HCC according to phase III placebo-controlled trial $^{[14]}$.

The use of sorafenib in the adjuvant setting was evaluated in the phase III STORM trial. In this study, 1114 patients who had undergone complete resection or ablation were randomized to sorafenib $400 \mathrm{mg}$ twice daily or placebo for up to four years. According to the final analysis, no difference in recurrence-free survival between the groups was demonstrated, with a median to time to recurrence of 33.3 months in the sorafenib group and 33.7 months in the placebo group $(\mathrm{HR}=0.94, P=0.26)^{[15]}$. Thus, the positive results obtained with sorafenib in advanced disease have not been reproduced in the adjuvant context post resection or ablation. A possible explanation for the negative result of the STORM trial is the absence risk stratification in the inclusion criteria, defining patients at high risk of recurrence. Additionally, adverse events of sorafenib (diarrhea, dermatological reactions, fatigue, etc.) which determined dose reduction or early therapy discontinuation may have impaired efficacy. 
In addition to sorafenib, other agents are approved for the treatment of advanced stage disease. Lenvatinib was shown to be non-inferior to sorafenib in a phase III trial ${ }^{[16]}$. Cabozantinib, regorafenib, and ramucirumab demonstrated improved overall survival over placebo in sorafenib-experienced patients with advanced $\mathrm{HCC}^{[17]}$. The successes with drugs in advanced-stage disease often prime studies focused on the translation into early stages of the disease in the neoadjuvant and adjuvant settings. However, after the negative results of the STORM trial, none of these drugs have been evaluated in patients after resection or ablation.

\section{Chemotherapy and radiotherapy}

The use of cytotoxic chemotherapy such as capecitabine or tegafur/uracil has been studied in the adjuvant setting for HCC patients, but no robust results have been reported yet ${ }^{[18]}$. Accordingly, the use of external radiation therapy has not shown significant benefit in the post-resection setting. Controversial data indicate a potential benefit from the use of intra-arterial injection of iodine-131, but more data are need in such modalities $^{[19]}$.

\section{Adjuvant immunotherapy}

The biological background for the use of immunotherapy in HCC is based on the fact that the liver is an organ with a remarkable immunotolerance due to the high antigenic load derived from the enteral-portal circulation. Additionally, HCC develops in a microenvironment of chronic inflammation and underlying liver disease. Low infiltration of CD8+ T lymphocytes, responsible for the antitumor immune response, and a marked presence of exhausted lymphocytes and regulatory T lymphocytes are described in HCC, contributing to an immunosuppressive and procarcinogenic microenvironment ${ }^{[8]}$.

The HCC microenvironment is thought to play a key role in determining the risk of recurrence. A high composite score incorporating density of $\mathrm{CD} 8+, \mathrm{CD} 3+\mathrm{T}$ cells was associated with a reduced risk of recurrence ${ }^{[20]}$. In addition, trafficking of activated lymphocytes into the tumor is regulated by VEGF ${ }^{[21]}$. Gene sequencing can also be a tool to predict response to systemic treatment, such as immunotherapy and VEGFR-directed TKIs. Translational studies with a limited number of patients suggested that CTNNB1 mutations seem to be associated with resistance to immunotherapy, while mutations in $\mathrm{PI} 3 \mathrm{~K} / \mathrm{mTOR}$ pathway seems to indicate resistance to TKIs ${ }^{[22]}$.

In animal breast cancer models, local treatments such as surgery can modify tumor microenvironment towards immunosuppression, which can subsequently promote tumor proliferation and metastasis. Similarly, ablation can induce tumor antigen release and stimulate inflammatory and cytokine production within the treated site. Therefore, the rationale of adding immunotherapy post local treatments is warranted in $\mathrm{HCC}^{[23]}$.

Immunotherapy consists of different modalities that comprehend the use of tumor peptide-based vaccines, cell therapies (CART and TCR), cytokines-induced killer cells, and immune checkpoint inhibitors (antiPD1, anti-PDL1, and anti-CTLA4).

Regarding adjuvant treatment post-resection or ablation, there are few published studies and a growing number of ongoing studies.

Cytokine-induced killer cells consist of patient-harvested immune cells that are expanded ex vivo using cytokines cultures (interleukin-2) and anti-CD3 antibodies, resulting in a cell population with strong antitumor immune activity. 
Takayama et al. ${ }^{[24]}$ conducted a randomized study in which patients previously treated with surgical resection were allocated to receive adoptive autologous lymphocytes stimulated with interleukin-2 and antiCD3 antibody in five infusions for 6 months or observation. The experimental treatment resulted in a risk reduction of $18 \%$ in 4.4 years and a longer time to recurrence ( $48 \% v s .33 \%$ at three years). There was no difference in survival between the groups.

Lee et al. ${ }^{[25]}$ evaluated the use of this modality in a phase III randomized multicenter study that enrolled 230 patients with HCC treated with surgery, ablation, or percutaneous ethanol injection. Patients were randomized to receive a cytokine-induced cell injection 16 times for 60 weeks or observation. Recurrencefree survival was 44 months in the experimental group $v s .30$ months in the observation group [HR $=0.63$, (95\% confidence interval: 0.43-0.94)]. There was also a significant reduction in overall mortality and cancerspecific mortality. Adverse events were greater in the group that received treatment ( $62 \% v s .41 \%$ ), especially chills, pyrexia, and productive $\operatorname{cough}^{[25]}$.

A Chinese phase II study involving 46 patients showed favorable results with the use of an autologous tumor peptide vaccine after HCC resection, demonstrating an $81 \%$ reduction in recurrence risk ${ }^{[26]}$. Other studies with similar techniques have not reproduced the same results and neither cell therapies nor vaccines are used routinely.

In advanced stage HCC, therapies based on immune checkpoint inhibitors are well-established. The main drugs in this class are intended to block the PD1/PD-L1 interaction, which inhibits antitumor action of cytotoxic lymphocytes against tumor cells. The use of treatment combination based on atezolizumab (antiPDL1 antibody) and bevacizumab is considered to be the standard first-line treatment ${ }^{[27]}$. The combination of nivolumab (anti-PD1) and ipilimumab (anti-CTLA4) is also an option for the treatment of advanced HCC $^{[28]}$. Pembrolizumab (anti-PD1) produces durable response rates in up to $20 \%$ of patients with advanced disease $^{[29]}$.

Adjuvant therapies are often inspired by positive strategies in the setting of advanced disease. Both nivolumab (CHECKMATE-9DX trial) and pembrolizumab (KEYNOTE 937 trial) are being studied as adjuvant treatments in placebo-controlled trials.

Preliminary results of the NIVOLVE trial were presented in 2021. In this phase II single arm trial, 55 patients after surgery $(n=33)$ or ablation $(n=22)$ received nivolumab for 12 months. The median recurrence-free survival was 26 months. Grade 3-4 treatment-related adverse events were described in 18.9\% and adverse events leading to treatment withdraw occurred in $32.1 \%^{[30]}$. Table 1 shows the main ongoing phase III studies with immune checkpoint inhibitors in the adjuvant setting.

\section{Traditional Chinese medicine}

Developments in hepatocarcinogenesis knowledge provided opportunity to identify mechanisms whereby traditional Chinese medicine could improve tumor control and improve clinical outcomes. For example, Huaier is a wood mushroom that has been used for 1500 years in China and is suggested to induce cell cycle arrest at Go/G1 phase and inhibit angiogenesis ${ }^{[31]}$. A randomized trial with 1044 HCC patients showed that Huaier resulted in longer recurrence-free survival over placebo after surgical resection ${ }^{[32]}$. Another trial showed that traditional herbal medicine provided superior overall survival compared to TACE after HCC resection ${ }^{[33]}$. Due to the lack of multinational data and experience, this approach is not part of Western guidelines. 
Table 1. Main ongoing phase III studies with immune checkpoint inhibitors in the adjuvant setting

\begin{tabular}{|c|c|c|c|c|c|c|}
\hline Trial & Drug & Control & $n$ & $\begin{array}{l}\text { Local } \\
\text { treatment }\end{array}$ & $\begin{array}{l}\text { Expected } \\
\text { termination }\end{array}$ & Primary end-point \\
\hline $\begin{array}{l}\text { CHECKMATE } \\
\text { 9DX } \\
\text { (NCT03383458) }\end{array}$ & Nivolumab & Placebo & 530 & Surgery/ablation & 2025 & Recurrence-free survival \\
\hline $\begin{array}{l}\text { KEYNOTE } 937 \\
(\text { NCTO3867084) }\end{array}$ & Pembrolizumab & Placebo & 950 & Surgery/ablation & 2025 & $\begin{array}{l}\text { Recurrence-free survival and } \\
\text { overall survival }\end{array}$ \\
\hline $\begin{array}{l}\text { IMBRAVE } 050 \\
(\text { NCT04102098) }\end{array}$ & $\begin{array}{l}\text { Atezolizumab + } \\
\text { bevacizumab }\end{array}$ & $\begin{array}{l}\text { Active } \\
\text { surveillance }\end{array}$ & 662 & Surgery/ablation & 2027 & Recurrence-free survival \\
\hline $\begin{array}{l}\text { EMERALD-2 } \\
(\text { NCT03847428) }\end{array}$ & $\begin{array}{l}\text { Durvalumab + } \\
\text { bevacizumab }\end{array}$ & $\begin{array}{l}\text { Durvalumab + } \\
\text { placebo } \\
\text { Placebo + placebo }\end{array}$ & 888 & Surgery/ablation & 2024 & Recurrence-free survival \\
\hline
\end{tabular}

\section{NEOADJUVANT TREATMENT}

\section{Rationale for neoadjuvant treatment}

Hepatocellular carcinoma is an aggressive disease mostly diagnosed in advanced stages and only 15\%-20\% of tumors are deemed resectable upfront. Although negative margins are often observed at the time of resection, most patients present with tumor recurrence. It is hypothesized that recurrence is a result of persistent micrometastatic disease post-surgical resection. Neoadjuvant therapy could potentially improve outcomes for patients with HCC.

For the majority of solid tumors, neoadjuvant treatment strategies are tailored to tumor downstaging, elimination of micrometastatic disease, access tumor response in vivo, and provide conversion from unresectable to resectable tumor. Regarding HCC, a successful neoadjuvant treatment could enable parenchymal sparing liver resections, conserving liver function and improving outcomes.

\section{TACE}

TACE exerts its activity through the combination of arterial embolization and arterial chemotherapy infusion. In the case of HCC, this modality has a clear rationale, once the blood supply derives from the hepatic artery system ${ }^{[34]}$.

Most studies regarding neoadjuvant TACE published to date have conflicting results regarding outcomes such as overall survival, recurrence, and disease-free survival ${ }^{[35-37]}$. However, studies show that TACEinduced tumor necrosis might have a relevant impact on patient outcome. Results from a meta-analysis of 32 randomized and nonrandomized studies comparing preoperative TACE to resection show that preoperative TACE did not improve disease-free survival or overall survival. In this analysis, it was shown that complete response following TACE was associated with superior survival compared to resection alone. On the other hand, incomplete or no tumor necrosis did not impact positively in outcomes ${ }^{[38]}$.

Some patients may present with liver-only disease, but beyond criteria for resection. In these cases, neoadjuvant TACE can be an encouraging conversion strategy. In a cohort with 831 patients treated with TACE over a 10-year period, 82 patients were successfully downstaged and 43 underwent salvage surgery. Surgical resection provided longer median survival when compared to those who refused a salvage resection (49 months vs. 31 months, $P=0.027$ ). Among patients who had a complete response, no difference in survival was demonstrated (50 months vs. 54 months, $P=0.699$ ), but a difference was observed in the subgroup that had a partial response ( 49 months $v s .24$ months, $P<0.001$ ). Such findings suggest a critical role for resection following downstaging with TACE in patients with a partial respons ${ }^{[39]}$. 
TACE can implicate in severe post-embolization syndrome and severe inflammation leading to adhesions. Therefore, current evidence is insufficient to determine which patients will develop a satisfactory response and, thus, would be an adequate candidate for such therapeutic approach.

\section{Transarterial radioembolization}

Transarterial radioembolization (TARE) is an intraarterial modality that uses microspheres loaded with radioactive isotopes, such as Ytrium-90 ${ }^{[40]}$. TARE is associated with a lower risk of post-embolization syndrome compared to TACE. Besides, TARE can induce hypertrophy of the contralateral future liver remnant, being an important tool in potentially resectable disease ${ }^{[41]}$.

Interesting results were reported from the LEGACY study, a multicenter, single-arm, retrospective study that included patients with solitary HCC $\leq 8 \mathrm{~cm}$. Among the 162 patients included, TARE served as neoadjuvant therapy for transplantation or resection in $21.0 \%$ (34 of 162) and 6.8\% (11 of 162) of patients, respectively, and as primary treatment for all others. Three-year overall survival was $92.8 \%$ for those neoadjuvant patients with resected or transplanted liver ${ }^{[42]}$.

Although most guidelines do not recommend TARE for downstaging, an expert consensus group suggested that TARE can be a potential bridging/downstaging therapy ${ }^{[43]}$. Therefore, while further research is warranted, including prospective clinical trials, neoadjuvant TARE with Y-90 may be appropriate for patients with advanced HCC who require downstaging for resection.

\section{Chemotherapy}

Direct delivery of chemotherapy into hepatic arteries can maximize chemotherapy delivery to liver tumors and minimize systemic toxic effects due to first-pass liver metabolism. A randomized controlled phase III trial randomly assigned patients with resectable BCLC stage A/B HCC beyond Milan criteria before hepatectomy to receive either neoadjuvant transarterial infusion chemotherapy (TAI) with FOLFOX or operation directly without any neoadjuvant treatment. The one-, two-, and three-year survival rates for TAI group were $92.9 \%, 78.6 \%$, and $63.5 \%$, respectively, and they were $79.5 \%, 62.0 \%$, and $46.3 \%$ for the group that underwent direct surgical resection, respectively. In addition, the 6-, 12-, and 18-month PFS rates for TAI group were $77.6 \%, 50.4 \%$, and $47.4 \%$, respectively, and they were $52.7 \%, 42.8 \%$, and $34.8 \%$ for patients who did not receive neoadjuvant treatment, respectively. The overall survival and recurrence-free survival were significantly better in the neoadjuvant treatment group $(P=0.016 \text { and } 0.017 \text {, respectively })^{[44]}$.

\section{Ongoing trials}

A phase IIa open label study presented the results of neoadjuvant cemiplimab, an anti-PD-L1 (NCT03916627). In this study, significant tumor necrosis, defined as greater than $70 \%$ necrosis of the resected tumor, was reported in 4 of 20 patients following treatment with neoadjuvant cemiplimab, meeting the primary end point of the study. Additionally, $15 \%$ of patients $(n=3 / 20)$ achieved complete tumor necrosis of $100 \%$, while $35 \%$ of patients $(n=7 / 20)$ had tumor necrosis of at least $50 \%{ }^{[45]}$.

More recently, the preliminary results of a two-part, multicenter, phase Ib study (NCT03682276) designed to assess safety and bioactivity of nivolumab plus ipilimumab combination prior to liver resection in earlystage HCC were reported. Of the seven patients enrolled, ORR in efficacy-evaluable patients $(n=5)$ was $20 \%$, including one partial response and four disease stabilizations. Pathological responses were observed in three out of five pathologically evaluable patients $(60 \%)^{[46]}$. Other ongoing studies involving neoadjuvant treatment approaches to HCC treatment are reported in Table 2. 
Table 2. Main ongoing studies with immune checkpoint inhibitors in the neoadjuvant setting

\begin{tabular}{|c|c|c|c|c|c|c|}
\hline & NCT03222076 & AURORA/NCT03337841 & NCT03510871 & $\begin{array}{l}\text { PRIME- } \\
\text { HCC/NCT03682276 }\end{array}$ & NCT04123379 & NCT03916627 \\
\hline $\begin{array}{l}\text { Patient } \\
\text { population }\end{array}$ & $\begin{array}{l}n=45 \\
\text { - Prior therapy allowed, including } \\
\text { surgery, RT, LRT, and systemic } \\
\text { therapy (sorafenib and } \\
\text { chemotherapy) } \\
\cdot \text { PS } \leq 1\end{array}$ & $\begin{array}{l}n=50 \\
\cdot \text { Child-Pugh A } \\
\cdot \text { PS } 0\end{array}$ & $\begin{array}{l}n=40 \\
\cdot \text { HCC with potential for } \\
\text { curative surgical } \\
\text { resection } \\
\text { - Prior local therapy } \\
\text { allowed } \\
- \text { ECOG PS } 0 \text { or } 1 \\
- \text { Child-Pugh A }\end{array}$ & $\begin{array}{l}n=32 \\
\cdot \text { HCC, ineligible for liver } \\
\text { transplant } \\
\cdot \text { PS } 0 \text { or } 1 \\
\cdot \text { Child-Pugh A }\end{array}$ & $\begin{array}{l}n=50 \\
\cdot \text { PS } 0 \text { or } 1\end{array}$ & $\begin{array}{l}n=94 \\
\cdot \text { PS } 0 \text { or } 1\end{array}$ \\
\hline Treatment & $\begin{array}{l}\text { Nivolumab q2w } \pm \text { ipililumab q2w } \times 3 \\
\text { doses } \rightarrow \text { liver surgery } \rightarrow \text { nivolumab } \\
\mathrm{q} 4 \mathrm{w} \pm \text { ipililumab q6w }\end{array}$ & $\begin{array}{l}\text { Pembrolizumb } 200 \mathrm{mg} \times 1 \text { dose } \rightarrow \\
\text { resection or RFA } \rightarrow \text { Pembrolizumb } 200 \\
\text { mg q3w }\end{array}$ & $\begin{array}{l}\text { Nivolumab + Ipilimumb } \\
\rightarrow \text { curative surgery, if } \\
\text { eligible }\end{array}$ & $\begin{array}{l}\text { Nivolumab } 3 \mathrm{mg} / \mathrm{kgq} 3 \mathrm{w} \times 2+ \\
\text { Ipilimumab } 1 \mathrm{mg} / \mathrm{kg} \times 1 \text { dose }\end{array}$ & $\begin{array}{l}\text { Nivolumab } 3 \mathrm{mg} / \mathrm{kg} \mathrm{q} 3 \mathrm{w} \times 2 \pm \\
(\text { BMS- } 813160 \text { or BMS-986253) } \rightarrow \\
\text { surgery } \rightarrow \text { nivolumab q4 } \times 3 \times 3\end{array}$ & Cemiplimab \\
\hline $\begin{array}{l}\text { Primary } \\
\text { endpoint(s) }\end{array}$ & Safety & 1-year RFS & Tumor shrinkage & Delay to surgery, safety & $\begin{array}{l}\text { Major pathologic response, } \\
\text { significant tumor necrosis }\end{array}$ & $\begin{array}{l}\text { Significant tumor } \\
\text { necrosis }\end{array}$ \\
\hline
\end{tabular}

RT: Radiotherapy; LRT: locoregional therapies; PS: performance status; RFA: radiofrequency ablation.

\section{CHALLENGES IN TRIAL DESIGN}

The interpretation of adjuvant studies for HCC is complex due to several factors related to patient selection. Patients undergoing surgery, ablation, or transplantation have different risk stratifications for recurrence. In general, studies include different risk profiles, depending on tumor size, number of nodules, histological differentiation, and microvascular invasion. It is necessary to develop stratification tools that standardize risk prediction, as well as the future incorporation of tools for molecular tumor characterization.

Atezolizumab (anti-PD-L1) plus bevacizumab (anti-VEGF) combination is the standard of care for patients with advanced stage disease ${ }^{[27]}$. After progression to first line, TKIs such as sorafenib and lenvatinib are the most adopted options in clinical practice. Other alternatives such as regorafenib, cabozantinib, and ramucirumab are reasonable choices in later lines, whenever patients present well preserved liver function and performance status ${ }^{[1]}$. Some of these agents are being tested in the (neo)adjuvant setting. Whether these drugs will retain their activity in both early stage and after recurrence requires further exploration. Positive results in these trials would influence decision making on how to treat recurrences and advanced stage HCC.

Finally, safety is a key challenge for clinical trials in early stages. HCC patients are at risk for clinical deterioration due to the underlying liver disease. Some drugs are associated with treatment-related adverse events that can lead to liver decompensations and figure as a competing risk of morbimortality. 


\section{CONCLUSION}

This article presents promising data regarding the future of neoadjuvant and adjuvant therapeutic approaches, with a potential benefit for a large number of patients with HCC who present a significant risk of recurrence and death after curative-intended treatment. The impact of an effective (neo)adjuvant treatment is more relevant in the natural history of HCC than the treatment of disease at later stages, as it has the potential to increase cure rates rather than prolong survival in patients with incurable disease.

Immunotherapy-based therapeutic choices are emerging as a topic of interest. It is important to highlight that clinical and molecular risk factors of recurrence are needed to selectively stratify candidates for (neo)adjuvant treatment. Furthermore, surrogates of response to systemic treatments in patients with HCC are warranted in order to better identify target patient populations who may benefit from these approaches. Ongoing trials have the potential to further change the current understanding and practice of resectable tumors in patients with HCC in the near future.

\section{DECLARATIONS}

\section{Authors' contributions}

Substantial contributions to conception and design of the study and performed data analysis, writing and interpretation: Mathias-Machado MC

Substantial contributions to conception and design of the study and performed data analysis, writing and interpretation: Fonseca L

\section{Availability of data and materials}

Not applicable.

\section{Financial support and sponsorship}

None.

\section{Conflicts of interest}

Both authors declared that there are no conflicts of interest.

\section{Ethical approval and consent to participate}

Not applicable.

\section{Consent for publication}

Not applicable.

\section{Copyright}

(c) The Author(s) 2021.

\section{REFERENCES}

1. Association for the Study of the Liver. Electronic address: easloffice@easloffice.eu, European Association for the Study of the Liver. EASL clinical practice guidelines: management of hepatocellular carcinoma. J Hepatol 2018;69:182-236. DOI

2. Hasegawa K, Kokudo N, Makuuchi M, et al. Comparison of resection and ablation for hepatocellular carcinoma: a cohort study based on a Japanese nationwide survey. J Hepatol 2013;58:724-9. DOI PubMed

3. Heimbach JK, Kulik LM, Finn RS, et al. AASLD guidelines for the treatment of hepatocellular carcinoma. Hepatology 2018;67:35880. DOI PubMed

4. Portolani N, Coniglio A, Ghidoni S, et al. Early and late recurrence after liver resection for hepatocellular carcinoma: prognostic and therapeutic implications. Ann Surg 2006;243:229-35. DOI PubMed PMC

5. Ouyang L, Lee J, Park CK, et al. Whole-genome sequencing of matched primary and metastatic hepatocellular carcinomas. BMC Med Genomics 2014;7:2. DOI PubMed PMC

6. Choi JH, Kim MJ, Park YK, et al. Mutations acquired by hepatocellular carcinoma recurrence give rise to an aggressive phenotype. 
Oncotarget 2017;8:22903-16. DOI PubMed PMC

7. Okajima W, Komatsu S, Ichikawa D, et al. Liquid biopsy in patients with hepatocellular carcinoma: circulating tumor cells and cellfree nucleic acids. World J Gastroenterol 2017;23:5650-68. DOI PubMed PMC

8. Ma J, Zheng B, Goswami S, et al. $\mathrm{PD} 1{ }^{\mathrm{Hi}} \mathrm{CD}^{+} \mathrm{T}$ cells correlate with exhausted signature and poor clinical outcome in hepatocellular carcinoma. J Immunother Cancer 2019;7:331. DOI PubMed PMC

9. Sun HC, Tang ZY, Wang L, et al. Postoperative interferon alpha treatment postponed recurrence and improved overall survival in patients after curative resection of HBV-related hepatocellular carcinoma: a randomized clinical trial. J Cancer Res Clin Oncol 2006;132:458-65. DOI PubMed

10. Zhang W, Song TQ, Zhang T, et al. Adjuvant interferon for early or late recurrence of hepatocellular carcinoma and mortality from hepatocellular carcinoma following curative treatment: a meta-analysis with comparison of different types of hepatitis. Mol Clin Oncol 2014;2:1125-34. DOI PubMed PMC

11. Yin J, Li N, Han Y, et al. Effect of antiviral treatment with nucleotide/nucleoside analogs on postoperative prognosis of hepatitis B virus-related hepatocellular carcinoma: a two-stage longitudinal clinical study. J Clin Oncol 2013;31:3647-55. DOI PubMed

12. Wang Z, Ren Z, Chen Y, et al. Adjuvant transarterial chemoembolization for HBV-related hepatocellular carcinoma after resection: a randomized controlled study. Clin Cancer Res 2018;24:2074-81. DOI PubMed

13. Wei W, Jian PE, Li SH, et al. Adjuvant transcatheter arterial chemoembolization after curative resection for hepatocellular carcinoma patients with solitary tumor and microvascular invasion: a randomized clinical trial of efficacy and safety. Cancer Commun (Lond) 2018;38:61. DOI PubMed PMC

14. Llovet JM, Ricci S, Mazzaferro V, et al; SHARP Investigators Study Group. Sorafenib in advanced hepatocellular carcinoma. $N$ Engl J Med 2008;359:378-90. DOI PubMed

15. Bruix J, Takayama T, Mazzaferro V, et al. Adjuvant sorafenib for hepatocellular carcinoma after resection or ablation (STORM): a phase 3, randomised, double-blind, placebo-controlled trial. Lancet Oncol 2015;16:1344-54. DOI PubMed

16. Kudo M, Finn RS, Qin S, et al. Lenvatinib versus sorafenib in first-line treatment of patients with unresectable hepatocellular carcinoma: a randomised phase 3 non-inferiority trial. Lancet 2018;391:1163-73. DOI PubMed

17. Bruix J, da Fonseca LG, Reig M. Insights into the success and failure of systemic therapy for hepatocellular carcinoma. Nat Rev Gastroenterol Hepatol 2019;16:617-30. DOI PubMed

18. Xia Y, Qiu Y, Li J, et al. Adjuvant therapy with capecitabine postpones recurrence of hepatocellular carcinoma after curative resection: a randomized controlled trial. Ann Surg Oncol 2010;17:3137-44. DOI PubMed

19. Chen K, Xia Y, Wang H, Xiao F, Xiang G, Shen F. Adjuvant iodine-125 brachytherapy for hepatocellular carcinoma after complete hepatectomy: a randomized controlled trial. PLoS One 2013;8:e57397. DOI PubMed PMC

20. Gabrielson A, Wu Y, Wang H, et al. Intratumoral CD3 and CD8 T-cell densities associated with relapse-free survival in HCC. Cancer Immunol Res 2016;4:419-30. DOI PubMed PMC

21. Chen DS, Hurwitz H. Combinations of bevacizumab with cancer immunotherapy. Cancer J 2018;24:193-204. DOI PubMed

22. von Felden J, Craig AJ, Garcia-Lezana T, et al. Mutations in circulating tumor DNA predict primary resistance to systemic therapies in advanced hepatocellular carcinoma. Oncogene 2021;40:140-51. DOI PubMed

23. Brown ZJ, Greten TF, Heinrich B. Adjuvant treatment of hepatocellular carcinoma: prospect of immunotherapy. Hepatology 2019;70:1437-42. DOI PubMed

24. Takayama T, Sekine T, Makuuchi M, et al. Adoptive immunotherapy to lower postsurgical recurrence rates of hepatocellular carcinoma: a randomised trial. Lancet 2000;356:802-7. DOI PubMed

25. Lee JH, Tak WY, Lee Y, et al. Adjuvant immunotherapy with autologous dendritic cells for hepatocellular carcinoma, randomized phase II study. Oncoimmunology 2017;6:e1328335. DOI PubMed PMC

26. Kuang M, Peng BG, Lu MD, et al. Phase II randomized trial of autologous formalin-fixed tumor vaccine for postsurgical recurrence of hepatocellular carcinoma. Clin Cancer Res 2004;10:1574-9. DOI PubMed

27. Finn RS, Qin S, Ikeda M, et al; IMbrave150 Investigators. Atezolizumab plus bevacizumab in unresectable hepatocellular carcinoma. N Engl J Med 2020;382:1894-905. DOI PubMed

28. Yau T, Kang YK, Kim TY, et al. Efficacy and safety of nivolumab plus ipilimumab in patients with advanced hepatocellular carcinoma previously treated with sorafenib: The CheckMate 040 randomized clinical trial. JAMA Oncol 2020;6:e204564. DOI PubMed PMC

29. Zhu AX, Finn RS, Edeline J, et al. Pembrolizumab in patients with advanced hepatocellular carcinoma previously treated with sorafenib (KEYNOTE-224): a non-randomised, open-label phase 2 trial. Lancet Oncol 2018;19:940-52. DOI PubMed

30. Kudo M, Ueshima K, Nakahira S, et al. Adjuvant nivolumab for hepatocellular carcinoma (HCC) after surgical resection (SR) or radiofrequency ablation (RFA) (NIVOLVE): a phase 2 prospective multicenter single-arm trial and exploratory biomarker analysis. JCO 2021;39:4070. DOI

31. Liu J, Wang S, Zhang Y, Fan HT, Lin HS. Traditional Chinese medicine and cancer: History, present situation, and development. Thorac Cancer 2015;6:561-9. DOI PubMed PMC

32. Chen Q, Shu C, Laurence AD, et al. Effect of Huaier granule on recurrence after curative resection of HCC: a multicentre, randomised clinical trial. Gut 2018;67:2006-16. DOI PubMed

33. Zhai XF, Liu XL, Shen F, Fan J, Ling CQ. Traditional herbal medicine prevents postoperative recurrence of small hepatocellular carcinoma: a randomized controlled study. Cancer 2018;124:2161-8. DOI PubMed

34. Lencioni R, Petruzzi P, Crocetti L. Chemoembolization of hepatocellular carcinoma. Semin Intervent Radiol 2013;30:3-11. DOI PubMed PMC 
35. Jianyong L, Jinjing Z, Wentao W, et al. Preoperative transcatheter arterial chemoembolization for resectable hepatocellular carcinoma: a single center analysis. Ann Hepatol 2014;13:394-402. PubMed

36. Zhang Z, Wu M, Liu Q. The effect of preoperative transcatheter hepatic arterial chemoembolization on disease-free survival after hepatectomy for hepatocellular carcninoma. Zhonghua Zhong Liu Za Zhi 1999;21:214-6. PubMed

37. Monden M, Okamura J, Sakon M, et al. Significance of transcatheter chemoembolization combined with surgical resection for hepatocellular carcinomas. Cancer Chemother Pharmacol 1989;23 Suppl:S90-5. DOI PubMed

38. Qi X, Liu L, Wang D, Li H, Su C, Guo X. Hepatic resection alone versus in combination with pre- and post-operative transarterial chemoembolization for the treatment of hepatocellular carcinoma: a systematic review and meta-analysis. Oncotarget 2015;6:3683859. DOI PubMed PMC

39. Zhang Y, Huang G, Wang Y, et al. Is salvage liver resection necessary for initially unresectable hepatocellular carcinoma patients downstaged by transarterial chemoembolization? Oncologist 2016;21:1442-9. DOI PubMed PMC

40. Kallini JR, Gabr A, Salem R, Lewandowski RJ. Transarterial radioembolization with Yttrium-90 for the treatment of hepatocellular carcinoma. Adv Ther 2016;33:699-714. DOI PubMed PMC

41. Gaba RC, Lewandowski RJ, Kulik LM, et al. Radiation lobectomy: preliminary findings of hepatic volumetric response to lobar yttrium-90 radioembolization. Ann Surg Oncol 2009;16:1587-96. DOI PubMed

42. Salem R, Johnson GE, Kim E, et al. Yttrium-90 radioembolization for the treatment of solitary, unresectable HCC: the LEGACY study. Hepatology 2021. DOI PubMed

43. Schwarz RE, Abou-Alfa GK, Geschwind JF, Krishnan S, Salem R, Venook AP; American Hepato-Pancreato-Biliary Association; Society of Surgical Oncology; Society for Surgery of the Alimentary Tract. Nonoperative therapies for combined modality treatment of hepatocellular cancer: expert consensus statement. HPB (Oxford) 2010;12:313-20. DOI PubMed PMC

44. Li S, Zhong C, Li Q, et al. Neoadjuvant transarterial infusion chemotherapy with FOLFOX could improve outcomes of resectable BCLC stage A/B hepatocellular carcinoma patients beyond Milan criteria: an interim analysis of a multi-center, phase 3, randomized, controlled clinical trial. JCO 2021;39:4008. DOI

45. Neoadjuvant cemiplimab demonstrates complete pathological responses in hepatocellular carcinoma [abstract]. Proceedings of the American Association for Cancer Research Annual Meeting 2021; 2021 Apr 10-15 and May 17-21. Philadelphia (PA): AACR; Cancer Res 2021;81(13_Suppl):Abstract nr CT182. DOI

46. Pinato DJ, Cortellini A, Pai M, et al. PRIME-HCC: Phase Ib study of neoadjuvant ipilimumab and nivolumab prior to liver resection for hepatocellular carcinoma. J Clin Oncol 2021;39:e16131. DOI PubMed PMC 